European

Thyroid Journal
Eur Thyroid J 2019;8:186-191

DOI: $10.1159 / 000497201$
Received: October 30, 2018

Accepted after revision: January 22, 2019

Published online: April 2, 2019

\title{
CA19-9 as a Predictor of Worse Clinical Outcome in Medullary Thyroid Carcinoma
}

\author{
Renata Alencar ${ }^{\mathrm{a}, \mathrm{b}}$ Daniel Barretto Kendler ${ }^{\mathrm{b}}$ Fernanda Andrade $^{\mathrm{a}}$ Carla Nava $^{\mathrm{a}}$ \\ Daniel Bulzico $^{a}$ Cencita Cordeiro de Noronha Pessoa ${ }^{a}$ Rossana Corbo ${ }^{a, b}$ \\ Fernanda Vaisman ${ }^{a, b}$ \\ aEndocrinology Service, Department of Medicine, Instituto Nacional do Cancer (INCA), Rio de Janeiro, Brazil; \\ ${ }^{b}$ Endocrinology Service, Department of Medicine, Hospital Universitário Clementino Fraga Filho, Rio de Janeiro, Brazil
}

\section{Keywords}

Medullary thyroid carcinoma - CA19-9. Calcitonin .

Prognostic factor

\begin{abstract}
Objective: Medullary thyroid carcinoma (MTC) is a rare disease, and its classic tumor marker is calcitonin. However, recently, very aggressive cases have been reported to also secrete carbohydrate antigen 19-9 (CA19-9), and its role as a marker of worse prognosis has been questioned. The aim of this study was to analyze the relationship between CA19-9 serum levels and MTC outcomes. Methods: We retrospectively reviewed 122 MTC patients followed in a tertiary cancer center from 1985 to 2017. Clinical-pathologic characteristics, therapeutic approaches, and outcomes were recorded and CA19-9 was collected. Results: Of the 122 patients included in the study, 48 had distant metastases, and at the end of follow-up $18.1 \%$ had structural persistent disease and $32.7 \%$ had progressive disease. CA19-9 was significantly higher in those who had disease progression than in those who had not (21.4 [14.3-110.9] vs. 7.27 [0.6-44.75] U/mL, $p=0.01$ ) and was also higher in patients who died from MTC (18.4 [14.3-110.9] vs. 7.59 [0.6-67.8] $\mathrm{U} / \mathrm{mL}, p<0.001)$. Fur-
\end{abstract}

thermore, using a ROC curve analysis, the cutoff point for CA19-9 in MTC patients was lower than that observed in pancreatic tumors. Conclusion: CA19-9 might have a role as a prognostic factor in addition to calcitonin and carcinoembryonic antigen in metastatic MTC.

(C) 2019 European Thyroid Association Published by S. Karger AG, Basel

\section{Background}

Carbohydrate antigen 19-9 (CA19-9) or sialyl Lewis antigen is a well-established tumor marker for the followup of pancreatic tumors [1-3]. Its serum levels can be elevated in other malign/benign conditions of the digestive [2] and respiratory tracts [4], and it has been associated with some clinical cases of poor evaluation described in the literature, such as prostate cancer $[5,6]$ and papillary thyroid cancer with anaplastic transformation [7]. Moreover, it has been described as a recurrent disease marker in pulmonary cancer [8] and papillary thyroid cancer [9], among others.

Vierbuchen et al. [10] detected CA19-9 in 5\% of medullary thyroid carcinoma (MTC) tissues. In addition, recent

\section{KARGER}

E-Mail karger@karger.com

www.karger.com/etj (c) 2019 European Thyroid Association

Published by S. Karger AG, Basel
Fernanda Vaisman, MD, PhD

Endocrinology Service, Department of Medicine, Instituto Nacional do Cancer José Alencar Gomes da Silva, INCA, HC 1

Praça da Cruz Vermelha, 23, Centro, Rio de Janeiro, RJ 20231-083 (Brazil)

E-Mail fevaisman@globo.com 
studies observed an increase in the serum levels of CA19-9 in clinical cases of aggressive MTC presentation [11, 12], suggesting that it can be a marker of bad prognosis in these cases. Milman et al. [12] described the case of a 56-year-old woman diagnosed with MTC at the age of 9 years (multiple endocrine neoplasia type 2B [MEN 2B]) who presented with extensive metastases to the lungs and liver 47 years after the diagnosis, followed by clinical deterioration and death, with high serum levels of CA19-9 (39,334 U/mL). Later, Elisei et al. [11] published the case of a 28-year-old man who was diagnosed with MTC after bone lesion biopsy, with multiple metastases. He died 10 months after the initial diagnosis and presented with an elevated concentration of CA19-9 at the time of clinical deterioration that increased from 5,248 to $>10,000 \mathrm{U} / \mathrm{mL}$. In this case, immunohistochemistry was performed and showed a strong immunoreactivity for CA19-9 in liver and pulmonary metastases.

The literature about this field is still very scarce, and only few studies can be found. It is not clear which is the responsible mechanism for this event, but one possible mechanism is the increase of CA19-9 expression caused by hypoxia-resistant cells with a high degree of malignancy, which become the predominant clones in advanced tumors with a high frequency of metastases [1].

The aim of this study was to evaluate the behavior of CA19-9 levels in patients with MTC followed at the Brazilian National Institute of Cancer (INCA) and to correlate its levels with the clinical presentation of the disease.

\section{Material and Methods}

Study Design and Population

This study examined a retrospective cohort of 122 patients diagnosed with MTC from 1985 to 2017 and followed at INCA's Endocrine Oncology Unit, a reference center for MTC treatment. All patients with an MTC diagnosis followed up at INCA during the period mentioned above were included, both sporadic and familial, except if they had pancreatic adenocarcinoma, colon cancer, biliary tract cancer, liver disease, gall bladder disease, inflammatory bowel disease, or any other malignant/benign disease in the digestive tract.

Most patients underwent surgical treatment with total or partial thyroidectomy and were monitored after for serum levels of calcitonin and carcinoembryonic antigen (CEA) usually twice or three times a year. After 2015, CA19-9 serum levels were also routinely measured in these patients and were collected usually at each semester. Imaging tests were done according to each case, i.e., neck ultrasound and cross-sectional imaging.

Medical records were assessed, and a questionnaire was filled in with the following variables: (a) age, (b) gender, (c) size, (d) presence of local and distant metastases, (e) presence of RET pro-

CA19-9 as a Prognostic Marker for

Medullary Thyroid Carcinoma
Table 1. Characteristics of the population $(n=122)$

\begin{tabular}{|c|c|}
\hline \multicolumn{2}{|l|}{ Age, years } \\
\hline Mean \pm SD & $46.8 \pm 16.4$ \\
\hline Median (range) & $46(12.1-87.9)$ \\
\hline \multicolumn{2}{|l|}{ Sex, $n(\%)$} \\
\hline Female & $77(63.1)$ \\
\hline Male & $45(36.9)$ \\
\hline \multicolumn{2}{|l|}{ Size, $\mathrm{cm}$} \\
\hline Mean \pm SD & $3.1 \pm 2.0$ \\
\hline Median (range) & $2.5(0.2-9.0)$ \\
\hline \multicolumn{2}{|l|}{$\mathrm{T}, n(\%)$} \\
\hline $\mathrm{T} 1$ & $26(21.3)$ \\
\hline $\mathrm{T} 2$ & $16(13.1)$ \\
\hline $\mathrm{T} 3$ & $37(30.3)$ \\
\hline $\mathrm{T} 4$ & $17(14)$ \\
\hline $\mathrm{Tx}$ & $26(21.3)$ \\
\hline \multicolumn{2}{|l|}{$\mathrm{N}, n(\%)$} \\
\hline N0 & $37(30.3)$ \\
\hline N1a & $12(9.8)$ \\
\hline N1b & $66(54.1)$ \\
\hline $\mathrm{Nx}$ & $7(5.7)$ \\
\hline Distant metastases, $n(\%)$ & 48 \\
\hline Lung & $22(18)$ \\
\hline Liver & $22(18)$ \\
\hline Bone & $28(10.6)$ \\
\hline Others & $7(5.8)$ \\
\hline RET mutation positive & $23(18.9)$ \\
\hline \multicolumn{2}{|l|}{ Median first postoperative calcitonin } \\
\hline (range), $\mathrm{pg} / \mathrm{mL}$ & $92(<2-23,367)$ \\
\hline \multicolumn{2}{|l|}{ Median first postoperative CEA } \\
\hline \multicolumn{2}{|l|}{ Median first postoperative CA19-9 } \\
\hline (range), $\mathrm{U} / \mathrm{mL}^{\mathrm{a}}$ & $7.85(0.6-110.9)$ \\
\hline \multicolumn{2}{|l|}{ Response to initial therapy, $n(\%)$} \\
\hline Excellent & $21(17.2)$ \\
\hline Biochemical incomplete & $46(37.7)$ \\
\hline Structural incomplete & $46(37.7)$ \\
\hline Not sufficient data & $9(7.4)$ \\
\hline \multicolumn{2}{|l|}{ Final status, $n(\%)$} \\
\hline No evidence of disease & $33(27.1)$ \\
\hline Biochemical persistent & $27(22.1)$ \\
\hline Structural persistent & $22(18.1)$ \\
\hline Progressive disease & $40(32.7)$ \\
\hline Disease-related death, $n(\%)$ & $37(30.3)$ \\
\hline Median follow-up (range), months & $74(3-395)$ \\
\hline
\end{tabular}

${ }^{\mathrm{a}} n=65$.

to-oncogene mutation, (f) first postoperative CEA, calcitonin, and CA19-9, (g) response to initial therapy, and (h) final status.

\section{Outcomes}

Response to therapy was based on the definitions cited by Lindsey et al. [13] in 2015 for MTC: (a) excellent response - undetect- 
Table 2. Factors associated with progressive disease at any time during follow-up: univariate analysis

\begin{tabular}{lccc}
\hline & Progression $(n=40)$ & No progression $(n=78)$ & $p$ value \\
\hline Age, years & $58.8(47-66)$ & $44.4(12.1-87.9)$ & 0.02 \\
Size, cm & $3.9(0.7-9.0)$ & $2.0(0.2-8.3)$ & 0.01 \\
N1 & $34(85)$ & $42(53.8)$ & $<0.001$ \\
M1 & $36(90)$ & $12(15.3)$ & $<0.001$ \\
RET mutation positive & $5(12.5)$ & $8(9.1)$ & 0.6 \\
First postoperative calcitonin, pg/mL $(n=65)$ & $409(70-23,367)$ & $43(<2-4,526)$ & 0.05 \\
First postoperative CEA, ng/mL & $77.43(3.02-1,923)$ & $4.6(0.56-220)$ & 0.01 \\
First postoperative CA19-9, U/mL & $21.4(14.3-110.9)$ & $7.27(0.6-44.75)$ & 0.01 \\
Follow-up, months & $59.5(3-396)$ & $90.4(6-352)$ & 0.01 \\
\hline
\end{tabular}

Values are medians (ranges) or $n(\%)$. Five patients were lost to follow-up.

Table 3. Factors associated with disease-specific mortality

\begin{tabular}{lccc}
\hline & Dead $(n=37)$ & Alive $(n=80)$ & $p$ value \\
\hline Age, years & $46.6(18.9-87.9)$ & $45.6(12.1-81.4)$ & 0.26 \\
Size, cm & $3.9(0.7-9.0)$ & $2.6(0.2-8.3)$ & 0.01 \\
N1 & $32(86.4)$ & $44(55)$ & 0.001 \\
M1 & $32(86.4)$ & $16(20)$ & $<0.001$ \\
RET mutation positive & $19(51.3)$ & $50(62.5)$ & 0.09 \\
First postoperative calcitonin, pg/mL & $407(70-23,367)$ & $42.5(<2-4,526)$ & 0.01 \\
First postoperative CEA, ng/mL & $77.43(3.02-1,923)$ & $4.6(0.56-220)$ & 0.01 \\
First postoperative CA19-9, $\mathrm{U} / \mathrm{mL}(n=65)$ & $18.4(14.3-110.9)$ & $7.59(0.6-67.8)$ & $<0.001$ \\
Follow-up, months & $53.1(6-314)$ & $98.8(3-396)$ & $<0.001$ \\
\hline
\end{tabular}

Values are medians (ranges) or $n(\%)$. Five patients were lost to follow-up.

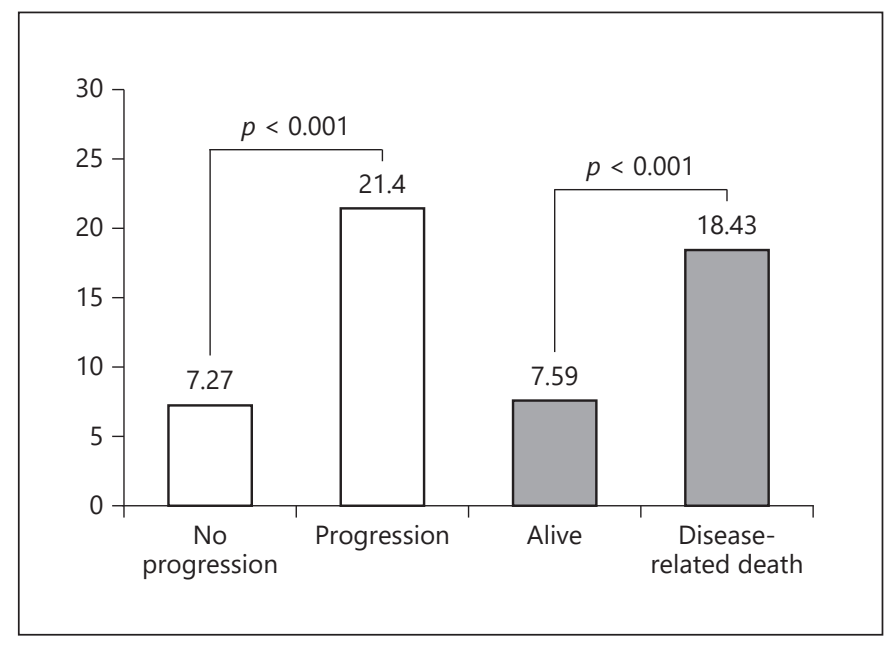

Fig. 1. Median first postoperative CA19-9 level in different groups. able calcitonin and normal CEA without structural evidence of disease; (b) biochemical incomplete response - detectable calcitonin or abnormal CEA in the absence of structural evidence of disease; and (c) structural incomplete response - persistent/recurrent structural disease regardless of calcitonin and CEA.

To determine the final status, at the end of the follow-up period the patients were categorized as (a) free of disease - undetectable calcitonin and normal CEA, with no evidence of structural disease; (b) biochemical persistent disease - detectable calcitonin or abnormal CEA, with no evidence of structural disease; (c) structural persistent disease - evidence of persistent/recurrent structural disease on cross-sectional imaging of functional studies, regardless of the biochemical status; (d) progressive disease - progression evidence of the disease based on RECIST criteria version 1.1 - a $20 \%$ or greater increase in the sum of the longest diameter of measured lesions (target lesions), taking as a reference the smallest sum largest diameter recorded since the treatment started or the appearance of one or more new lesions; and (e) death related to the disease - patient died because of the progression of the disease. 
CA19-9, Calcitonin, and CEA

Biochemical evaluation was done by the same laboratory for CA19-9 using an electrochemiluminescence immunoassay (Elecsys, Cobas), with the normal range $<37 \mathrm{U} / \mathrm{mL}$. The calcitonin measurement was performed using a radioimmunoassay from 1998 to 2003 with the normal range between 23 and 71 pg/mL. After 2004, the measurement was done by chemiluminescence, with a reference value $<11.5 \mathrm{pg} / \mathrm{mL}$ and functional sensitivity $<2 \mathrm{pg} / \mathrm{mL}$. CEA serum levels were measured by an electrochemiluminescence assay, and values $<5 \mathrm{ng} / \mathrm{mL}$ were considered normal.

\section{Statistical Analysis}

Analyses were performed using SPSS system statistical software for MAC version 20. For univariate analyses, the following methods were used: for comparisons with numerical data, either Student's $t$ test (parametric) for independent samples or the MannWhitney test (nonparametric) was used. To identify the independent variables that predict an excellent response to initial therapy or no evidence of disease at the final status, logistical regression analysis was used. A ROC curve was used to establish an optimal cutoff for CA19-9, and Kaplan-Meyer survival curves with logrank analysis were also employed after that. A $p$ value $<0.05$ was considered significant.

\section{Results}

This study included 122 patients, mostly women (77 females and 45 males), with a median age of 46 (12.1$87.9)$ years and a median follow-up period of $74(3-395)$ months. The first postoperative CA19-9 $(n=65)$ was 7.85 $\mathrm{U} / \mathrm{mL}$, calcitonin was $92 \mathrm{pg} / \mathrm{mL}$, and CEA was $7.82 \mathrm{ng} /$ $\mathrm{mL}$. A total of $37.7 \%$ of the patients was considered as having a structural incomplete response, while at the end of follow-up $18.1 \%$ had structural persistent disease and $32.7 \%$ had progressive disease, with $30.3 \%$ disease-related deaths. Table 1 shows other relevant clinical parameters of the sample.

A univariate analysis was performed to establish which factors were associated with progressive disease at any time during the follow-up (Table 2). In this study, patients that had progressive disease had a higher first postoperative CA19-9 (21.4 [14.3-110.9] vs. 7.27 [0.6-44.75] $\mathrm{U} / \mathrm{mL}, p=0.01$ ), were older at diagnosis (54.8 vs. 44.4 years), had larger tumors as well as more frequently metastatic disease and higher calcitonin levels. The presence of a RET mutation showed no significance in this analysis.

In Table 3, factors associated with disease-related death are listed, and CA19-9 was also a significant factor. Patients who died from the disease presented with higher levels of CA19-9 at diagnosis. Tumor size, the presence of local or distant metastases, and first postoperative calci-

CA19-9 as a Prognostic Marker for

Medullary Thyroid Carcinoma

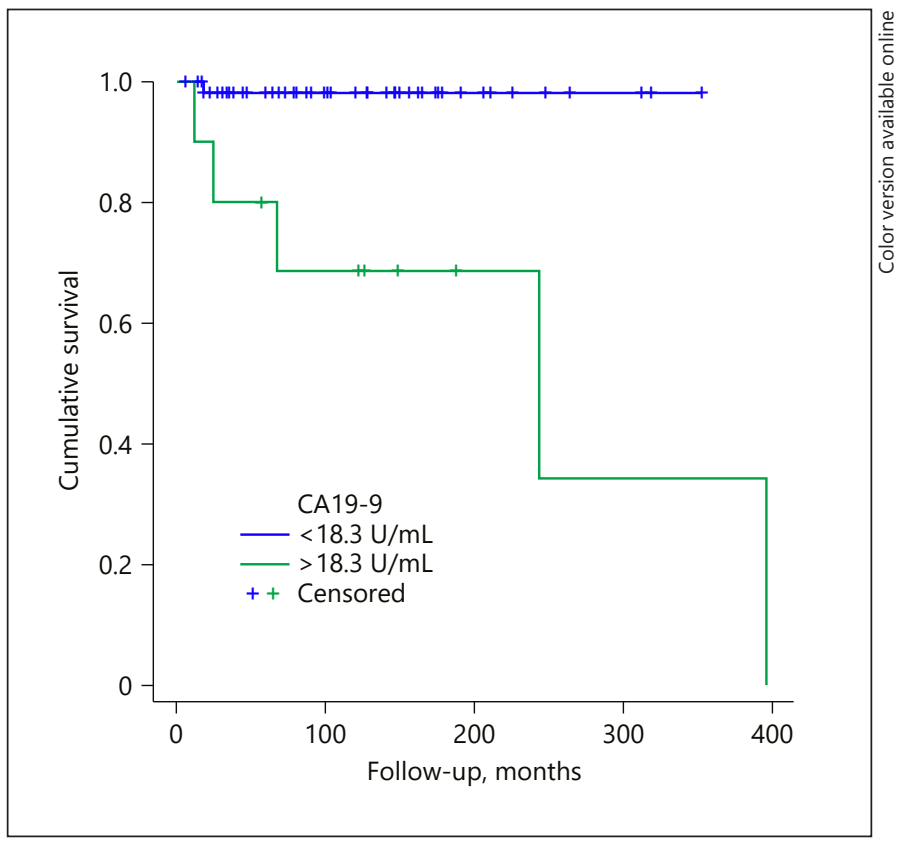

Fig. 2. OS according to postoperative CA19-9 levels (group 1: $<18.3 \mathrm{U} / \mathrm{mL}$, OS 345 [333-358] months vs. group 2: >18.3 U/mL, OS 230 [106-354] months, $p<0.001)$. OS, overall survival.

tonin and CEA also reached the level of statistical significance. Surprisingly, age at diagnosis was not a significant factor associated with death in this group.

Figure 1 illustrates the comparison of serum levels of CA19-9 between the groups with progressive disease and stable disease and between the alive group and the disease-related death group, showing the significance of this tumor maker in the groups with a worse outcome. However, CA19-9 doubling time did not show statistical significance with regard to progression or disease-related death. First postoperative calcitonin showed a linear correlation with first postoperative CA19-9 $\left(R^{2}=11.9, p=\right.$ 0.01 ).

Using a ROC curve analysis, a cutoff for CA19-9 of $18.3 \mathrm{U} / \mathrm{mL}$ had a sensitivity of $83 \%$ and a specificity of $91 \%$ (area under the curve $0.937,95 \%$ CI $0.87-0.99, p<$ 0.01 ) in predicting progression and disease-related death. Using this cutoff, Figure 2 shows the correlation with a shorter overall survival (OS) when CA19-9 was $>18.3$ $\mathrm{U} / \mathrm{mL}(<18.3 \mathrm{U} / \mathrm{mL}$ : OS 345 [333-358] months vs. $>18.3$ $\mathrm{U} / \mathrm{mL}$ : OS 230 [106-354] months, $p<0.001)$.

Among the 65 patients who had CA19-9 measured, the median time between surgery and the first measurement of CA19-9 was $85.5(1-375)$ months. The association between the time CA19-9 was measured after surgery and 
prognosis was not statistically significant. The same prognostic factors were significant in this specific group: presence of lymph node and distant metastases, first calcitonin after surgery, and the higher the CA19-9 the higher was the risk of bad outcomes.

\section{Discussion}

The present study explores the association between CA19-9, usually produced by pancreatic and epithelial cells, and MTC prognosis. In our study, we were able to show that postoperative serum levels of CA19-9 tend to be higher at the beginning of follow-up in patients more likely to progress and to die from MTC. In our cohort, the best cutoff to predict bad outcomes was $18.3 \mathrm{U} / \mathrm{mL}$. Interestingly, a linear correlation between postoperative levels of CA19-9 and bad outcomes was found; however, we were not able to find a significant correlation using its doubling time, maybe because it is thought to be a marker of less differentiated tumors and is not related with volume and proliferation of disease. Furthermore, we also found a linear correlation between first postoperative calcitonin and first postoperative CA19-9.

Although the literature on this issue is scarce, our findings are in accordance with most of the existing studies. Elisei et al. [14] also showed that an elevated level of serum CA19-9 could be a predictor of mortality independent of calcitonin doubling time. It was also observed in this study that $16 \%$ of the advanced structural recurrent/persistent disease group had higher levels of CA19-9 and had a higher mortality rate than the control group which had a normal level of this marker ( 68.7 vs. $23.8 \%$, respectively, $p=0.0004$ ). In addition, they found an association between serum CA19-9 levels and the presence of bone and liver metastases, and positivity of this marker was an important predictor of mortality independent of calcitonin doubling time. Furthermore, in agreement with our results, Elisei et al. [14] also found a linear correlation between CA19-9, calcitonin, and CEA. In the same study, they were able to show that most of the patients who had abnormal serum CA19-9 also showed positive cytoplasmic staining for this protein in the primary tumor, which corroborates our hypothesis that it is produced by the primary tumor and no other possible source. Like CEA, CA19-9 in this scenario could be regarded as a marker of less differentiation as it is a molecule produced by epithelial cells [14].
Milman et al. [15] explored the correlation between CA19-9 staining of MTC tissue and its metastatic potential and they found that $100 \%$ of the primary specimens that were documented to have metastatic spread stained positive for CA19-9. Luo et al. [16] explored the role of serum CA19-9 levels as a prognostic biomarker for pancreatic neuroendocrine tumors, and they demonstrated that levels $>16 \mathrm{U} / \mathrm{mL}$ are associated with poor prognosis, advanced stage, and aggressive characteristics. This cutoff value was similar to the one found in our study and suggests that CA19-9 might be a prognostic factor of bad outcome for neuroendocrine tumors in general, not only for MTC.

Some other significant findings in our study were the risk factors for disease-specific mortality, such as tumor size, local and distant metastases, RET mutation, and first postoperative calcitonin, CEA, and CA19-9. Some factors are already well established in the literature [13, 17]. Kuo et al. [18] found that age, tumor size $>2 \mathrm{~cm}$, and local and distant metastases are risk factors associated with disease-specific mortality. Kebebew et al. [19] also described age and TNM stage of disease as independent predictors of survival. Momin et al. [20] showed that increasing tumor size and neck metastasis are poor prognostic factors. Surprisingly, age in our sample was not associated with disease-related death but only with progressive disease, while we could observe exactly the opposite about the RET mutation, even though it is known that some kinds of RET mutation can lead to an aggressive clinical condition as observed by Wells et al. [17]. Progressive disease, tumor size, local and distant metastases, and first postoperative calcitonin, CEA, and CA19-9 were also significant factors in our analysis.

As this was a retrospective study, we are aware of some limitations, such as the lack of standardization to assess progression, the fact that some patients could have been lost to follow-up, and that not all patients had their CA19-9 measured as this was not part of our routine in the past. However, even with those limitations we were able to show a very strong correlation with prognosis.

Furthermore, CA19-9 is widely available in our country, even in more places than calcitonin, and is also cheaper to measure; thus, its possible use as a surrogate marker in MTC could be interesting. Also, when calcitonin doubling time is indeterminate, as it is between 6 and 24 months, CA19-9 could provide additional prognostic information. 
In conclusion, this study shows that CA19-9 tends to be higher in patients with a worse prognosis even within the "reference range." In the present study, the cutoff for CA199 was much lower than the one traditionally used for pancreatic tumors, suggesting that maybe, for MTC, a specific "reference range" should be established. In fact, the markers could be combined to give a more accurate long-term prognosis and tailor initial therapy and follow-up.

\section{Statement of Ethics}

The subjects enrolled in the study have given their written informed consent. The study protocol has been approved by the research institute's committee on human research.

\section{Disclosure Statement}

No competing financial interests exist for any of the authors.

\section{References}

1 Usón Junior PLS, Callegaro-Filho D, Bugano DDG, Moura F, Maluf FC. Predictive value of serum carbohydrate antigen 19-9 (CA19-9) for early mortality in advanced pancreatic cancer. J Gastrointest Cancer. 2018 Dec;49(4):481486.

2 Galli C, Basso D, Plebani M. CA 19-9: handle with care. Clin Chem Lab Med. 2013 Jul; 51(7):1369-83

3 Ballehaninna UK, Chamberlain RS. Serum CA $19-9$ as a biomarker for pancreatic cancer - A comprehensive review. Indian J Surg Oncol. 2011 Jun;2(2):88-100.

4 Kim HR, Lee CH, Kim YW, Han SK, Shim YS, Yim JJ. Increased CA 19-9 level in patients without malignant disease. Clin Chem Lab Med. 2009;47(6):750-4.

5 Yokoyama S, Fukuhara S, Imazu T, Hara T, Yamaguchi S, Adachi S. [Recrudescence of prostate cancer with low serum level of PSA and high serum level of CEA and CA19-9: a case report]. Hinyokika Kiyo. 2007 Jul;53(7):485-7.

6 Momma T, Kimura S, Saito S, Onoda N. [Prostate cancer with high serum level of CEA and CA19-9: a case report]. Hinyokika Kiyo. 1998 Mar;44(3):187-91.

7 Ogawa M, Hori H, Hirayama M, Kobayashi M, Shiraishi T, Watanabe Y, et al. Anaplastic transformation from papillary thyroid carcinoma with increased serum CA19-9. Pediatr Blood Cancer. 2005 Jul;45(1):64-7.
8 Isaksson S, Jönsson P, Monsef N, Brunnström $\mathrm{H}$, Bendahl PO, Jönsson M, et al. CA 19-9 and CA 125 as potential predictors of disease recurrence in resectable lung adenocarcinoma. PLoS One. 2017 Oct;12(10):e0186284.

9 Yamaguchi E, Makino Y, Sato T, Uchida M, Harada Y, Maruyama R. CA19-9-producing lung metastasis after surgery for papillary thyroid carcinoma: report of a case. Surg Today. 2014 Nov;44(11):2157-61.

10 Vierbuchen M, Larena A, Schröder S, Hanisch FG, Ortmann M, Larena A, et al. Blood group antigen expression in medullary carcinoma of the thyroid. An immunohistochemical study on the occurrence of type 1 chain-derived antigens. Virchows Arch B Cell Pathol Incl Mol Pathol. 1992;62(2):79-88.

11 Elisei R, Lorusso L, Romei C, Bottici V, Mazzeo S, Giani C, et al. Medullary thyroid cancer secreting carbohydrate antigen 19-9 (Ca 19-9): a fatal case report. J Clin Endocrinol Metab. 2013 Sep;98(9):3550-4.

12 Milman S, Whitney KD, Fleischer N. Metastatic medullary thyroid cancer presenting with elevated levels of CA 19-9 and CA 125. Thyroid. 2011 Aug;21(8):913-6.

13 Lindsey SC, Ganly I, Palmer F, Tuttle RM. Response to initial therapy predicts clinical outcomes in medullary thyroid cancer. Thyroid. 2015 Feb;25(2):242-9.

14 Elisei R, Lorusso L, Piaggi P, Torregrossa L, Pellegrini G, Molinaro E, et al. Elevated level of serum carbohydrate antigen 19.9 as predictor of mortality in patients with advanced medullary thyroid cancer. Eur J Endocrinol. 2015 Sep;173(3):297-304.
15 Milman S, Arnold JL, Price M, Negassa A, Surks MI, Fleischer N, et al. Medullary thyroid cancer that stains negative for CA 19-9 has decreased metastatic potential. Endocr Pract. 2015 Jun;21(6):590-4.

16 Luo G, Jin K, Cheng H, Liu C, Guo M, Lu Y, et al. Carbohydrate antigen 19-9 as a prognostic biomarker in pancreatic neuroendocrine tumors. Oncol Lett. 2017 Dec;14(6):6795800.

17 Wells SA Jr, Asa SL, Dralle H, Elisei R, Evans DB, Gagel RF, et al.; American Thyroid Association Guidelines Task Force on Medullary Thyroid Carcinoma. Revised American Thyroid Association guidelines for the management of medullary thyroid carcinoma. Thyroid. 2015 Jun;25(6):567-610.

18 Kuo EJ, Sho S, Li N, Zanocco KA, Yeh MW, Livhits MJ. Risk factors associated with reoperation and disease-specific mortality in patients with medullary thyroid carcinoma. JAMA Surg. 2018 Jan;153(1):52-9.

19 Kebebew E, Ituarte PH, Siperstein AE, Duh QY, Clark OH. Medullary thyroid carcinoma: clinical characteristics, treatment, prognostic factors, and a comparison of staging systems. Cancer. 2000 Mar;88(5):1139-48.

20 Momin S, Chute D, Burkey B, Scharpf J. Prognostic variables affecting primary treatment outcome for medullary thyroid cancer. Endocr Pract. 2017 Sep;23(9):1053-8. 